\title{
Acute geriatrics at the front door 䇺
}

\author{
Authors: Simon Conroy ${ }^{A}$ and Stuart Parker ${ }^{B}$
}

Older people with frailty and urgent care needs are major uses of health and social care services. Comprehensive geriatric assessment (CGA) is an evidence-based approach to improving their outcomes, as well as improving service outcomes. Geriatricians form a small proportion of the overall workforce and cannot address the population need alone, so all clinicians (doctors, nurses, therapists and so on) need to engage in delivering CGA as a process of care, underpinned by specific competencies - which can be developed. Delivery of this care pathway needs to be measured and improved as rigorously as campaigns like those for improving sepsis or eradicating methicillin-resistant Staphylococcus aureus.

\section{Introduction}

For many older people - in the UK at least - the emergency department (ED) is the main portal of entry to urgent care. In many cases, the older person's urgent care needs can be quickly assessed and managed within a short period of time (the 4-hour standard); in the UK, about one-third of people over the age of 85 years accessing EDs are able to return home directly. But for many others, a more detailed assessment and management plan is required, delivered in either observation wards in the ED (typically for people with an anticipated stay of less than 24 hours; variously termed clinical decision units, emergency decision units and other such acronyms) or an acute medical unit (for post-ED management of medical patients, initially for up to 72 hours) - staffed by acute physicians trained in acute assessment of a wide range of conditions. ${ }^{1}$

While older people (65 or $75+$ years) form a significant proportion of patients at all of these axes of care, and around two-thirds of all those receiving ongoing care in acute hospitals, there is a discrete subset of people aged $75+$ who account for the bulk of bed days. Emerging data from the Nuffield Trust indicate about $20 \%$ of all those aged $75+$ account for $85 \%$ of bed days and $85 \%$ of readmission or deaths within 90 days of an urgent care episode. It appears that this $20 \%$ resemble older people with frailty.

Frailty is a distinctive late-life health state in which apparently minor stressor events are associated with adverse health outcomes. The two established international models are the

Authors: ${ }^{A}$ professor of geriatric medicine, Leicester Royal Infirmary, Leicester, UK; ${ }^{B}$ William Leech professor of geriatric medicine, Newcastle University, Newcastle upon Tyne, UK frailty phenotype ${ }^{2}$ and the cumulative deficit model, ${ }^{3}$ both of which have been validated in large population cohort studies. The models identify people at increased risk of a range of adverse outcomes, including dependency, institutionalisation and premature death. Frailty can be measured; the cumulative deficit model of frailty expresses frailty as a ratio of deficits present to a total list of deficits that have been pre-defined using a standard procedure. ${ }^{4}$ This model maps well onto the Clinical Frailty Scale, ${ }^{5}$ which is quick ( $<1$ minute), simple and easy to use in urgent care settings. ${ }^{6}$

Frailty identification is important at several levels. Older people with frailty will often present with so-called nonspecific presentations, multiple comorbidities, functional decline and differential challenge - a constellation that challenges the traditional paradigms of urgent care responses. Non-specific presentation is a term that is often used to describe the recognisable and quite specific geriatric clinical syndromes, which include reduced mobility and immobility ('off legs'), falls, delirium ('confusion') and incontinence. In these presentations, the textbook clues for the specific underlying medical diagnoses may not be prominent. The

\section{Key points}

There are many older people accessing acute medical units, but not all older people are the same; frailty can be used to identify those who are at the greatest risk of harms, including long hospital stays

Comprehensive geriatric assessment is an evidence-based approach to the care of older people that can help clinicians form a holistic, patient-centred management plan that addresses what matters to the patient more than what is the matter with the patient

While many clinicians already work within multidisciplinary teams in acute care settings, communication and coordination in real time is critical

Effective care of older people with urgent care needs requires specific geriatric competencies, which can be learnt and applied by all clinicians

KEYWORDS: Acute care, comprehensive geriatric assessment, frailty 
presence of one of these geriatric clinical syndromes should prompt clinicians to focus on objective pointers towards an underlying diagnosis.

Frailty helps differentiate older people into risk groups with different outcomes. Identifying an individual with severe frailty at the point they access urgent care immediately signals that the individual is at risk of major harm - for example, an inpatient death rate of $30 \% .^{5}$ This might prompt a discussion, including the patient and their family/carer, to achieve a balance between interventional (eg high dependency unit/intensive care unit) and aggressive care (eg intravenous fluids and antibiotics) that address 'the matter with' patients versus a more palliative approach, in acknowledgement of the patient's and family wishes, that address 'what matters to' them. This will help clinical teams move from managing conditions to managing people. Aside from influencing clinical care, advantages of a frailty identification tool in secondary care include:

$>$ informing service provision

$>$ informing commissioning and reimbursement decisions

$>$ research and quality improvement.

\section{Interventions}

Comprehensive geriatric assessment $(\mathrm{CGA})^{7}$ offers an evidencebased structure to holistic assessment that is critical to improving outcomes for older people with frailty:

> medical - have you got a working primary diagnosis, as well as a list of comorbidities, which are active or important, that also require attention?

> psychological - have you assessed for the presence of delirium, dementia or depression/anxiety? These will have a substantial impact on ongoing management.

> functional ability - you may have made a diagnosis, but how will you get the patient 'clinically stable for transfer'. Being 'medically fit' is meaningless if the person cannot mobilise to the toilet and back safely.

> social circumstances - what support exists? What more is needed to enable a return home? Do you know how to access resources that can help?

> environment - is the home setting conducive to ongoing care needs, or are adaptations required? Do you know how to organise a home hazards review for people who have fallen?

CGA is used to guide treatment decision making in partnership with the patient, their carers and professional members of the multidisciplinary team. These decisions often need to take account of factors that affect the patient's home and care support arrangements. Carers are often under great personal strain and can be helped to continue supporting an older person in their own home if that strain is taken into account during assessment and decision making; for example, by the appropriate use of home care packages and the use of respite care facilities. Transfers of care and changes in living arrangements are often considered as part of a CGA. Judicious attention to carer strain and the complete knowledge of the patient's preferences and needs that is provided by CGA can help to reduce unnecessary or premature institutionalisation.

CGA is not yet a universally provided service in acute care in the UK, but is beginning to be more widely available than it used to be. Recent national survey work conducted by NHS
Benchmarking ${ }^{8}$ and others showed that in $2015-16$ over a third of hospital trusts had developed enhanced teams with geriatricians working in the ED. In addition, about half of trusts provide CGA in inpatient medical wards, EDs and decision units. In this survey, it was found that recognised assessment tools and pathways for frailty were present in most (59\%), but by no means all, of the local health and social care economies.

\section{Specific pitfalls in assessment and management}

Aside from the 'philosophy of care' (holistic assessment, values-driven decision making), the effective management of older people with frailty requires specific knowledge about presentations in in this group.

\section{Delirium and dementia}

Dementia and delirium are syndromes, not pathologies, and so the diagnosis is entirely dependent on the skill of health professionals. Routine assessment of cognition will identify moderate to severe cognitive impairment, but more subtle presentations can be missed. The 4-point Abbreviated Mental Test score (AMT-4) is quick to complete and has good correlation with the 10-point scale but is easier to apply requiring only place, age, date of birth and year. ${ }^{9}$ The detection of cognitive impairment should always be accompanied by an assessment for delirium, for example using the 4 -AT. ${ }^{10}$ Delirium has acute onset, typically over days and weeks. Failed detection is associated with a sevenfold hazard for increased mortality, ${ }^{11,12}$ and is an independent predictor of hospital length of stay. ${ }^{13}$ Symptoms may not only be cognitive, they may be behavioural, psychotic (hallucinations, delusions) or mood symptoms with little or absent signs of disorientation or cognitive impairment. For example, symptoms of depression in a delirious individual may initially appear indistinguishable from people suffering from depressive disorder. The key is to suspect delirium with any sudden change of mental state or behaviour in older people. The DSM 5 (Diagnostic and Statistical Manual of Mental Disorders, fifth edition) criteria for delirium, which also help distinguish this from dementia, are:

> disturbance in attention (ie reduced ability to direct, focus, sustain, and shift attention) and awareness

$>$ change in cognition (eg memory deficit, disorientation, language disturbance, perceptual disturbance) that is not better accounted for by a preexisting, established or evolving dementia

$>$ the disturbance develops over a short period (usually hours to days) and tends to fluctuate during the course of the day (information from carers or third parties is essential and will often hold the key)

> there is evidence from the history, physical examination or laboratory findings that the disturbance is caused by a direct physiologic consequence of a general medical condition, an intoxicating substance, medication use or more than one cause.

Both dementia and delirium impact on treatment, for example through raising questions about a person's capacity to make health and welfare decisions or practical issues such as concordance with therapies. 


\section{Falls and syncope}

Falls are the commonest single reason for older people to present to urgent care. Falls are not an inevitable part of ageing but are often due to underlying disease or impairment that may be amenable to treatment or modification.

Key in urgent care settings is to carefully differentiate between syncopal and non-syncopal falls; this is not always easy because of ante- and retrograde amnesia, which is common, or recall bias. All too often, direct witness accounts are not available, meaning that the clinician has to base their assessment of the balance of probabilities.

An understanding of cerebral perfusion pressure in older people is important, minor perturbations of which can result in (pre-)syncope. While national drivers promulgate a focus upon tight blood pressure (BP) control, there is ongoing debate about the ideal BP, which is linked to perfusion pressure in older people. ${ }^{14}$ Detailed discussion about $\mathrm{BP}$ control is beyond the scope of this article, but it is worth noting that blood pressures less than $120 \mathrm{mmHg}$ systolic in older people, especially where variation with lower troughs is evident, should prompt a careful examination of whether or not syncope is likely. It is useful to ask 'do you remember hitting the floor?' and not to accept vague assertions such as 'I must have tripped' as plausible explanations for the fall. The pattern of injury can also provide clues - facial bruising in particular is highly suspicious of syncope. The presence of syncope should prompt a review of medication and a search for underlying causes, at the very least a 12-lead electrocardiogram and routine bloods; other tests may be indicated.

\section{Polypharmacy}

While there has been a substantial focus on 'de-prescribing' in older people, driven by guidance such as the STOPP (screening tool of older people's prescriptions)-START (screening tool to alert to right treatment) criteria ${ }^{15}$ or the Anticholinergic Burden Scale, ${ }^{16}$ urgent care presentations do present an additional opportunity to review medication appropriateness. Consideration should be given as to where a patient is in their life - a consideration of trajectory, ${ }^{17}$ informed, when possible, by a description of physical and cognitive function over the last year. Some patients will be clearly entering a more palliative phase of life (consider the 'surprise question'), in which case, is it really helpful to continue antiplatelets or anticoagulants (side effects such as bruising, bleeding and low grade anaemia are common and their impact often under-estimated in older people with frailty)? Does someone who is bed-bound really require high-dose anti-anginals if they are no longer exerting themselves? Each de-prescribing scenario should consider the patients values or best interests formulations, the rationale for continuing the treatment (how will it help?) and the opportunity costs of continuing prescribing (remembering that time spent administering medication is time not spent on comfort care, for example). This can be a challenging task but, informed by guidance, practice and clinical supervision, could and arguably should be a routine part of urgent care assessments. Any changes and the rationale for the changes should be clearly communicated to primary care and carers.

\section{Outcomes}

Given the pressures that systems are under, there is a risk that in pursuit of metrics relevant to the operational perspective, patient benefit is neglected. Clinical teams should be asking 'what matters to the patient' - what is their preferred outcome from this episode of care? This might not relate to professional standards ('what is the matter with the patient' - eg exclusion of myocardial infarction), so much as concerns about loved ones or respecting preferences for comfort rather than cure. This information could be incorporated into the problem list, and might also lead to de-prioritising other aspects of care (such as investigations that the patient does not value).

\section{Summary and future vision}

Older people with frailty and urgent care needs are major uses of health and social care services. CGA is an evidence-based approach to improving their outcomes, as well as improving service outcomes. Geriatricians form a small proportion of the overall workforce, and cannot address the population need alone, so all clinicians (doctors, nurses, therapists and so on) need to engage in delivering CGA as a process of care, underpinned by specific competencies - which can be developed. Delivery of this care pathway needs to be measured and improved as rigorously as campaigns such as improving sepsis or eradicating methicillinresistant Staphylococcus aureus.

\section{Conflicts of interest}

The authors have no conflicts of interest to declare.

\section{References}

1 Percival F, Day N, Lambourne A, Derek B, Ward D. An evaluation of consultant input into acute medical admissions management in England, Wales and Northern Ireland. London: Royal College of Physicians, Society for Acute Medicine, 2010.

2 Fried L, Tangen C, Walston J et al. Frailty in Older adults: evidence for a phenotype. J Gerontol A Biol Sci Med Scie 2001;56:M146-56.

3 Rockwood K, Song X, MacKnight C et al. A global clinical measure of fitness and frailty in elderly people. CMAJ 2005;173:489-95.

4 Searle SD, Mitnitski A, Gahbauer E, Gill TM, Rockwood K. A standard procedure for creating a frailty index. BMC Geriatr, 2008;8:24.

5 Wallis SJ, Wall J, Biram RW, Romero-Ortuno R. Association of the clinical frailty scale with hospital outcomes. QJM 2015;108:943-9.

6 Elliott A, Phelps K, Regen E, Conroy SP. Identifying frailty in the emergency department-feasibility study. Age Ageing 2017 doi: 10.1093/ageing/afx089. [Epub ahead of print]

7 Ellis G, Whitehead MA, Robinson D, O’Neill D, Langhorne P. Comprehensive geriatric assessment for older adults admitted to hospital: meta-analysis of randomised controlled trials. BMJ 2011;343:d6553.

8 NHS Benchmarking Network. Older people in acute settings: benchmarking report. Manchester: NHS Benchmarking Network, 2016.

9 Swain DG, O’Brien AG, Nightingale PG. Cognitive assessment in elderly patients admitted to hospital: the relationship between the shortened version of the Abbreviated Mental Test and the Abbreviated Mental Test and Mini-Mental State Examination. Clin Rehabil 2000;14:608-10.

10 Bellelli G, Morandi A, Davis D et al. Validation of the 4AT, a new instrument for rapid delirium screening: a study in 234 hospitalised older people. Age Ageing 2014;43:496-502. 
11 Han J, Wilson A, Wesley E. Delirium in the older emergency department patient: a quiet epidemic. Emerg Med Clin North Am 2010;28:611-31.

12 Young J, Inouye SK. Delirium in older people. BMJ 2007;334:842-6.

13 Han JH, Zimmerman EE, Cutler N et al. Delirium in older emergency department patients: recognition, risk factors, and psychomotor subtypes. Acad Emerg Med 2009;16:193-200.

14 van der Wardt V. Should guidance for the use of antihypertensive medication in older people with frailty be different? Age Ageing 2015;44:912-3.

15 O’Mahony D, O’Sullivan D, Byrne S et al. STOPP/START criteria for potentially inappropriate prescribing in older people: version 2 . Age Ageing 2014;44:213-8.
16 Fox C, Richardson K, Maidment ID et al. Anticholinergic medication use and cognitive impairment in the older population: the medical research council cognitive function and ageing study. J Am Geriatr Soc 2011;59:1477-83.

17 Gill TM, Gahbauer EA, Han L, Allore HG. Trajectories of disability in the last year of life. N Engl J Med 2010;362:1173-80.

Address for correspondence: Dr Simon Conroy, Geriatric Medicine, Leicester Royal Infirmary, Infirmary Square, Leicester LE1 5WW, UK.

Email:spc3@le.ac.uk

\title{
Making difficult decisions with older patients on medical wards 洸结
}

\author{
Authors: Bushra Khizar ${ }^{\mathrm{A}}$ and Rowan $\mathrm{H}$ Harwood $^{\mathrm{B}}$
}

Decision making with older people can be difficult because of medical complexity, uncertainty (about prognosis, treatment effectiveness and priorities), difficulties brought by cognitive and communication impairment and the multiple family and other stakeholders who may need to be involved. The usual approach, based on balancing benefits and burdens of a treatment, and then deciding on the basis of autonomy (or best interests for someone lacking mental capacity), within the constraints of resources and equity, remains valid, but is often inadequate. In addition, approaches relying on optimal communication and relationship building and professional virtues are important. Older people vary in their medical status, views and preferences more than younger people and these variations must be sought and accommodated, using a shared decision-making approach. This includes adapting to the increasing numbers of people from different cultures.

\section{How do we decide?}

Scientific knowledge, the law, professional regulation, the organisations within which we work and the political and social environment all influence how we practise medicine. Quite apart from law and regulation, and sometimes in contravention to it, people have expectations about healthcare: who should be consulted over decisions and how we should approach death

Authors: ${ }^{\text {A }}$ specialist registrar, Nottingham University Hospitals NHS Trust, Nottingham, UK; ${ }^{B}$ consultant geriatrician, Nottingham University Hospitals NHS Trust, Nottingham, UK and dying. No single set of rules adequately covers all human activity, including medical practice, but ethical frameworks can help us analyse and understand difficult situations, and resolve or address them through better understanding.

\section{Key points}

Older people are medically complex, the information needed to make decisions is often uncertain and the impact of cognitive impairment can make decision making difficult

A systematic and thorough approach is required; the principlist approach of considering benefits, burdens, autonomy and justice can be applied, but principles often conflict

Open communication and relationship building with families and other stakeholders assumes greater importance

Individual variation must be identified, respected and accommodated in making decisions

Decisions at the end of life are open to the same general approach, but uncertainty in establishing prognosis and widespread overestimation of the efficacy of medical intervention can lead to over-intervention and a failure to adopt a palliative approach

KEYWORDS: Cognitive impairment, decision making, end-of-life care, ethics, older people, professional 